\title{
Surface Damage of Gold-Ion Implanted Co-WC Micro-Punch Tools during Press Processing
}

\author{
Shizuka Nakano ${ }^{\text {a) }}$, Ming Yang ${ }^{\text {b) }}$, Mikiko Yoshida ${ }^{\text {a) }}$, Hisato Ogiso ${ }^{\text {a) }}$ \\ a) National Institute of Advanced Industrial Science and Technology (AIST), \\ Namiki-1-2-1, Tsukuba, Ibaraki 305-8564, Japan \\ Fax: 029-861-7167 shizuka.nakano@aist.go.jp \\ Mikiko-yoshida@aist.go.jp \\ Ogiso.h@aist.go.jp \\ b) Tokyo Metropolitan University, Asahigaoka 6-6, Hino, Tokyo 191-0065, Japan \\ Fax: 042-585-8440, yang@tmu.ac.jp
}

\begin{abstract}
Surface damage of gold-ion implanted Co-WC micro-punch tools during press processing was evaluated by using a novel die system to monitor changes in the tool surface after each punch. A previously developed micro device fabrication system using micro press forming system was used to fabricate the tools. Despite the advantage of micro scale forming processes in downsizing production, the relative toughness of the formed tools is a serious problem. The lifespan of the tools, particularly punch tools, is too short for manufacturing use. Punch holes are typically smaller than $100 \mathrm{~mm}$, and the fracture toughness is equal to the stress. The lifespan of punch tools can be increased by using the ion implantation method that we previously developed, involving gold ion implantation with post thermal annealing. However the lifespan of ion implanted punch is not enough for manufacturing, we try for clear a reason of this achievement and try to increasing lifespan. In this current study, we used a novel die system to monitor the surface damage of a punch after each punch. Results revealed that adhesion of work material on the punch surface differed between the ion implanted and un-implanted surfaces of the punch tools. The surface modification decreased the adhesion of the sheet metals, thus increasing the lifespan of the punch tool.
\end{abstract}

\section{Introduction}

Press forming is a commonly used system to produce automobiles and electrical appliances, for example, because it is fast, inexpensive, and can combined with other processes such as punching, sharing, bending, drawing, and forging. Applications have expanded for micro-electro mechanical systems (MEMS), and other micro and nano devices, such as micro sensors, micro optical devices, and RF modules, while environmental concerns require conservation of energy and material in micro forming production, such as decreasing the diameter of the injection nozzles of automobile engines to increase energy efficiency. Expanding the industrial usage of micro devices requires a production system that is inexpensive and adaptable to various materials. Previously we developed a fabrication system for micro devices that uses a press forming system $[1,2]$. The downsizing of the production scale for micro devices generates two critical problems for press forming. First, stress in the press-formed tools increases with decreasing size of the tool; the force of the punch decreases with decreasing shearing length, whereas the cross-sectional area of the punch hole decreases as the square of the hole radius. Second, for a typical micro-punch tool (about $100 \mathrm{~mm}$ ), the toughness and stress in a press-formed tool are approximately the same. To improve the toughness of micro-punch tools, we previously applied an ion implantation method because the surface of the tool is related to friction between the tool and the sheet metal, hardness, and other mechanical properties $[3,4]$. In previous studies, $75 \mathrm{keV}$ gold ions were implanted into the side wall of punch tools at $1 \times 10^{16} \mathrm{~cm}^{-2}$ dose at room temperature, and then annealed at $700 \mathrm{~K}$ for $30 \mathrm{~min}$ in air. This process modifies the tool surface as follows. The gold ions fracture the Co-WC structure at the atomic level, thus changing the surface to amorphous. The post annealing re-crystallizes the surface via oxidization in which the implanted gold ions act as an oxidized catalyst. The surface of these tools is mainly $\mathrm{WO}_{3}$ poly-crystal. Compared with unmodified tools, such surface-modified tools have a lower friction coefficient, lower hardness. This surface modification increased the lifespan of the tools by a factor of 16 , compared with "un-implanted" tools. However, industry needs tools that have an even longer lifespan and that can be produced stably with various materials and process conditions. Therefore, the cause of damage to the tools and how to increase the lifespan of the tools need clarification. However, the tool is encircled by the die parts, and must remove from die to observe it surface. Direct observation of the interaction 
between the tool surface itself and the sheet metal will provide insight. In this current study, we therefore developed a die system to directly monitor a punch tool after each punch without having to remove the tool or sheet metal from the press machine. This new system, which we call microscopic wear-monitoring die system (MWM die system), involves a die system and a micro-scope video camera to capture photos of the tool surface after each punch. First, we monitored the change of surface of tools for 100 punches using this system and SEM-EDX. Then, we use the $\mathrm{WO}_{3}$ surface-modified tools and un-modified tools, Finally,
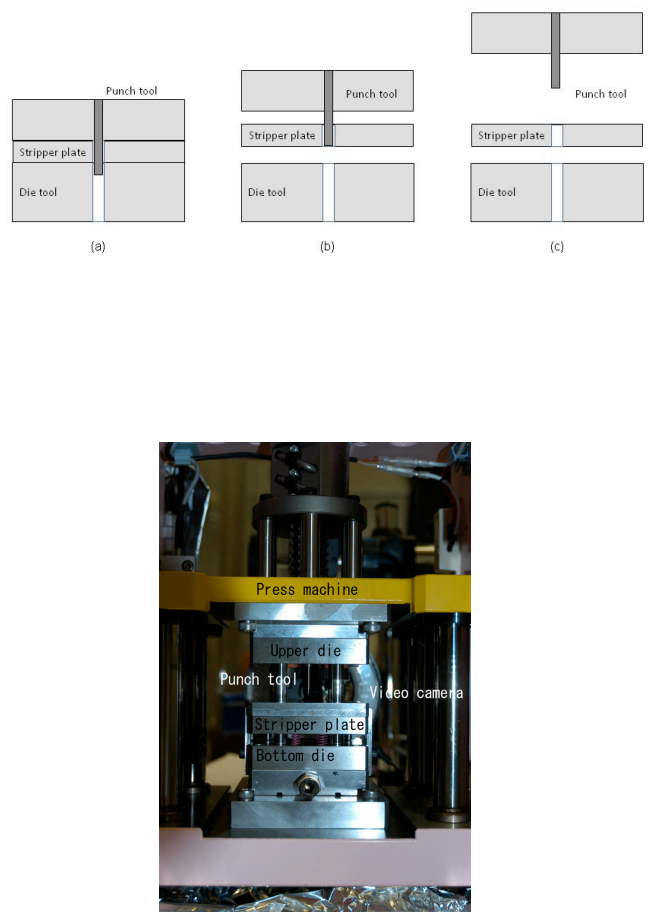

(d)

Figure 1 Schematic images and photograph of MWM die system. The die is move between (a) bottom and (b) upper position normally. Furthermore MWM die system move to (c) observation position. (d) Photograph of MWM die system of (c) observation position.

\section{Experimental procedure}

Figure 1 shows a photo of the MWM die system. A stripper plate is attached to an upper die that contains the punch tools, and thus "hides" the punch tools. By attaching a bottom die, the punch tool is visible when the upper die is raised (as shown in Fig. 1). A video microscope (Syouden-sya TG300PC) is used to photograph the punch tool after each punching shot. Figure 2 shows the video camera system and press machine set-up. The resolution of the video camera is $2048 \times 1536$; 1/2" type CMOS imaging system, with a microscope zoom lens of $\times 1.15$ to $\times 6.25$ with a minimum view area of about $2 \times 1.5 \mathrm{~mm}$.

To compare the effect of surface modification, we tested surface-modified and unmodified tools. Both tools were made from commercial Co-WC (Sumitomo
Electric Industries Ltd. XF1), which has a grain size 70 $\mathrm{nm}$ common in industrial usage for micro scale punch tools. (Note: This Co-WC material differs from that used in our previous studies [3,4], $0.5 \mathrm{~mm}$.) The surface modification was ion implantation combined with post thermal annealing that we developed previously[3,4]. First, $75 \mathrm{keV}$ gold ions were implanted at $1 \times 10^{16}$ ions $/ \mathrm{cm}^{2}$ dose at room temperature into the side-wall of the tool, which was rotated during implantation. Then, the tool was annealed at $700 \mathrm{~K}$ for 30 minutes in air. The reason of chosen this condition was also cleared in previous study, other some ion species could not increase life span, only this combination process had increased the lifespan[3]. However, the effects of this process are not cleared, and then we did this study.

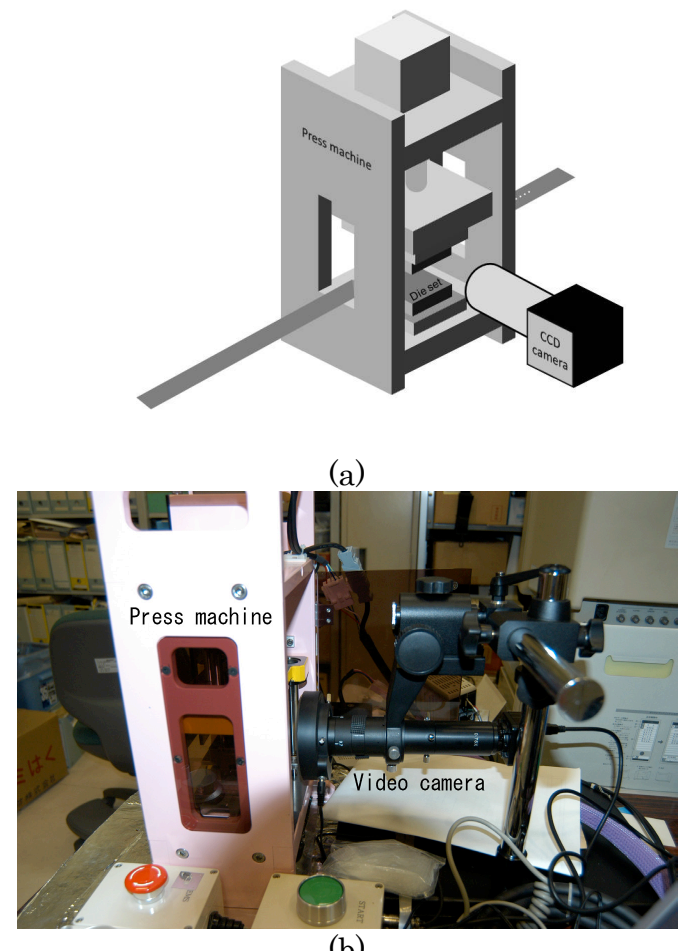

(b)

Figure 2 Schematic image and photograph of press system with CCD video camera.

Using this MWM die system, we compared the damage of punch tools with and without surface modification. The diameter of each punch hole was $150 \mathrm{~mm}$ and the sheet metal was 0.2 -mm-thick stainless spring steel (SUS-304CSP-1/2H). Each punch tool was used for 100 shots (in industrial usage, typically more than 10000 shots are used), using a screw servo press machine (Seki corporation Ltd., MS-10), which enabled the longest stroke possible for the upper die motion. The press speed was $30 \mathrm{~mm} / \mathrm{sec}$ and the press cycle was about $30 \mathrm{shots} / \mathrm{min}$. The press machine was stopped at the top dead center point for $1.5 \mathrm{sec}$ to obtain a photo of the punch tool. After the 100 shots, the tool was removed from the die system and then observed and analyzed using scanning electron microscopy (SEM) with an energy dispersive X-ray spectroscopy (EDX) system (JEOL JSM-5500 \& Oxford Link-ISIS). 


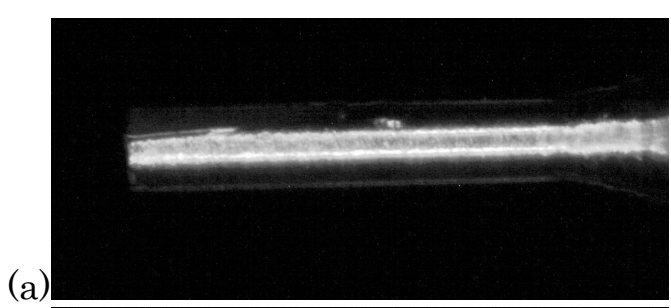

(a)

(b)

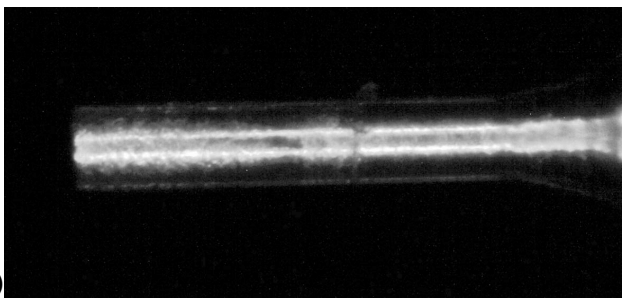

(c)

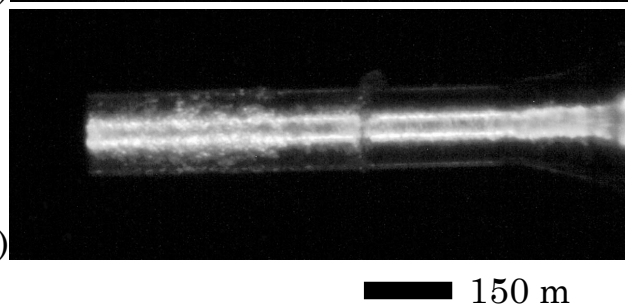

Figure 3 Images of surface-modified punch tools (a) before press test, (b) after 50 shots, and (c) after 100 shots. Surface modification was gold ion implantation combined with post annealing.

(a)

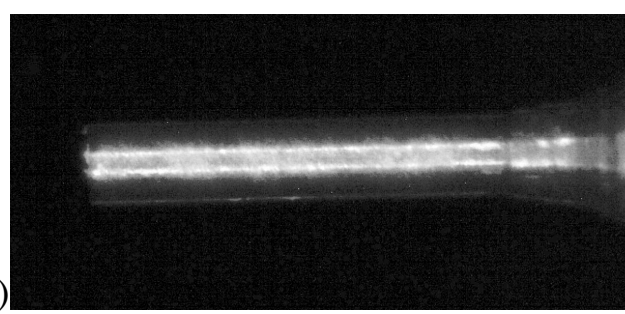

(b)

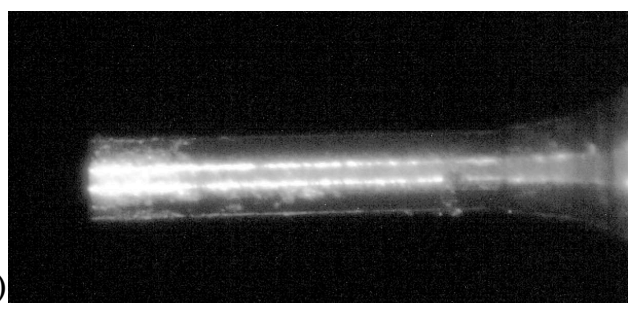

(c)

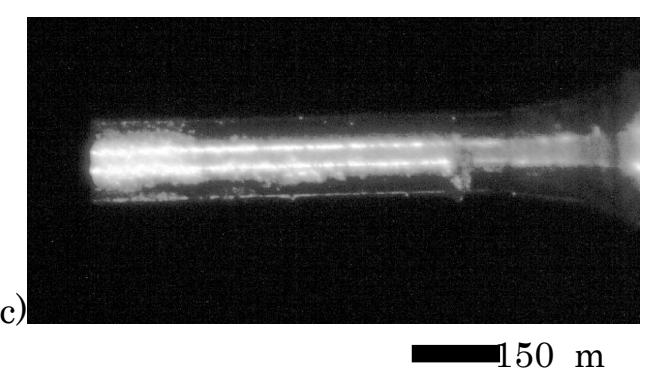

Figure 4 Images of surface-modified punch tools (a) before press test, (b) after 50 shots and (c) after 100 shots. Surface modification was gold ion implantation combined with post annealing.

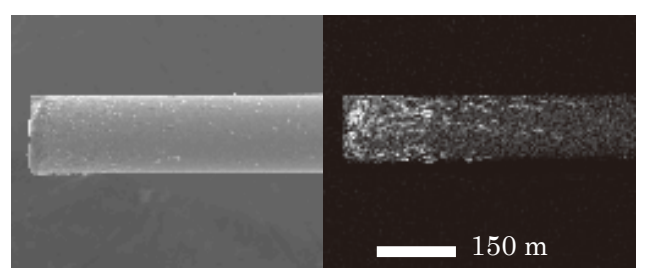

(a)

(b)

Figure 5 (a) SEM image and (b) Fe mapping by EDX image of surface-modified punch tool. Surface modification was gold ion implantation combined with post annealing.

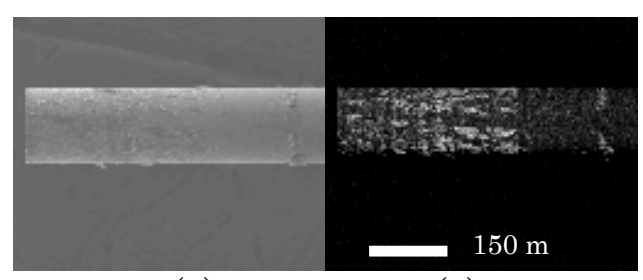

(a)

(b)

Figure 7 SEM image and (b) Fe mapping by EDX image of an unmodified punch tool.

\section{Results and discussion}

Figures 3 and 4 show representative images of unmodified and surface-modified punch tools, respectively, before and after 50 and 100 punches, sequentially revealing the change in surface damage. The damaged areas appear as lighter parts of the tool surface due to diffused reflection, and expand with increasing number of shots. The MWM die system and microscopic video camera system clearly reveals the surface condition of the punch tool. The bright areas in the image are due to reflection of light to the video camera from only about $1 / 3$ of the area of the tool. For both the unmodified and surface-modified tools, the surface before the punch test was smooth, and light reflection was only slight, and the surface became increasingly rougher with increasing shots. The diffused reflection region increased (Figs. 3a and 4a) with increasing punch shots and the surface became rougher (Figs.3b,c and $4 b, c)$. The diffused reflection was related to surface wear, i.e. roughness or adhesion. The diffused reflection region of the surface-modified tool was about half that of the unmodified tool, indicating that the surface modification deterred surface damage. For both punch tools, the area of surface damage after 100 shots was more severe than after 50 shots (Figs. $3 \mathrm{~b}, \mathrm{c}$ and $4 \mathrm{~b}, \mathrm{c})$. The unmodified punch tool showed larger damaged area expanded to its end of inserted point (Figs 3b,c). A large score was also found at the right side of figs $3 b, c$, which is same point of the end of inserted point into sheet metal.

Figure 5 shows an SEM image and EDX mapping image for Fe and Fig. 6 shows the EDX spectra of part of the $\mathrm{Fe}$ rich point of Fig.6 for the surface-modified tool after 100 shots. Adhesion of Fe and $\mathrm{Cr}$ from the sheet metal is clearly evident on the tools at the same region as the reflection region seen in 
the photos (Figs. 3 and 4). In the punch tests, the sheet metal was stainless steel constructed from $\mathrm{Fe}-\mathrm{Ni}-\mathrm{Cr}$, and the tool was only in contact with this sheet metal. Therefore, the tool damage of mainly parts were adhered stainless steel and it came from the sheet metal. Figure 7 shows SEM-EDX images for the unmodified tool. The results are similar to those for the surface-modified tool, namely, the relation between reflection region and adhesion region, although the reflection region is smaller for the surface-modified region. Our previous study showed that the lifespan of surface-modified punch tools is longer than that of unmodified tools $[3,4]$. The adhesion changes the size and shape of a tool, which in turn increases the resistance force in the pressing process and increases the stress in the tool. If the stress in a punch tool exceeds the toughness, the punch tool will fracture. If surface damage determines the lifespan of a punch tool due to adhesion from the sheet metal, then surface modification can deter adhesion and thus extend the lifespan of the tool. Despite the low number of shots in this study (only 100 shots) compared with the number in an actual production process, the surface modification involving gold ion implantation and annealing was able to decrease the area of adhesion damage. Surface modifications of press tools typically increase the hardness and/or decrease the friction efficiency [5-8]. Results from this current study show, however, that toughness is apparently affected not only by hardness or friction, but also by adhesion of the sheet metal on the tool surface itself. The interaction between a tool and the sheet metal is crucial in the lifespan of a tool. Future research should include using surface modification to minimize damage to a punch tool, thus extending its lifespan.

\section{Conclusion}

A MWM die system was developed to monitor the surface of punch tools. With this system and a microscopic camera, the tool surface after each punch can be monitored. Results revealed that the most serious damage is due to adhesion of sheet metal on the tool surface itself. These results were confirmed by SEM and EDX analysis. Results also revealed that surface modification involving gold ion implantation and annealing decreases this damage, indicating that the lifespan of punch tools is related to surface adhesion. More extensive testing is needed to clarify this relationship, however, because in this study, only 100 punches were done for each tool. Increased lifespan of tools requires further development of surface modification technology in which interaction between the tool and sheet metal is considered.

\section{References}

[1] M.Yang, S.Nakano, K.Manabe, K.Morikawa, K.Ito, H.Saito, K.Fuchigami, T.Yokoi, N.Hotta, Proc. 1st Int. Conf. On New Forming Technology, (2004), Harbin, 135-140

[2] K.Fujimoto, M.Yang, M.Hotta, H. Koyama, S.Nakano, K.Morikawa, J.Cairney, Journal of Materials Processing Technology, 177, 1-3, (2006), 639-643

[3] S.Nakano, K.Ito, M.Yang, H.Ogiso, Trans. Mat. Res. Soc. Jpn 32 (4) (2007) 865

[4] S. Nakano, K.Ito, M.Yang, M.Yoshida, H.Ogiso, Surf. Coat. Tech. 203 (2009) 2776

[5] A.D.Anderson, M.H.Loretto, G. Dearnaley, Mat. Sci. Eng. A105/106 (1988) 503-507

[6] J.-P. Hirvonen, J. Koskinen, A. Anttila, D. Stone, C. Paszkiet, Mat. Sci. Eng., 90 (1987) 343-347

[7] CHR. Weist, G. K. Wolf, P. Ballhause, Mat. Sci. Eng. 90 (1987) 399-405

[8] M.Takahashi, S.Nakano, H.Ogiso, T.Sano, S.Fuchizawa, J.Kagami, Proc. 1994 Japanese Spring Conf. for Tech. of Plasticity. (1994) Narahino, 79-82(in Japanese)

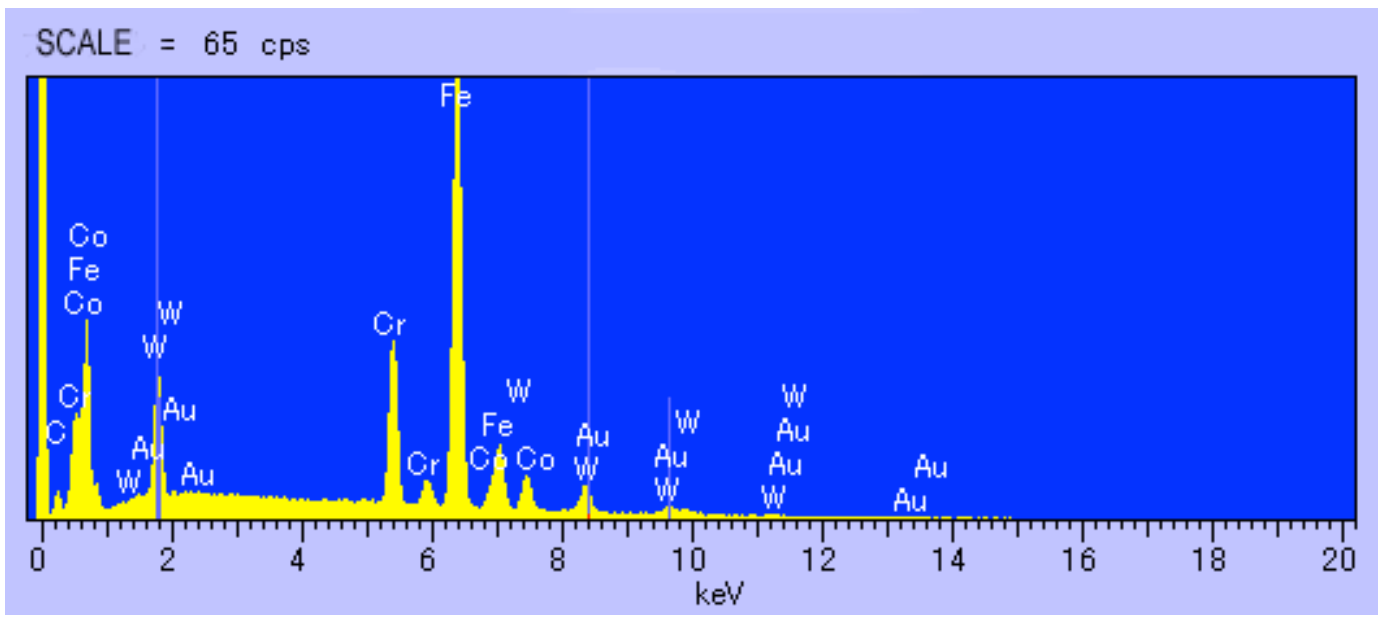

Figure 6 EDX spectra at adhesion point shown in Fig. 5. Fe and Cr peaks are clearly evident. 